\title{
Effective MKSVM Classifier with LDA Methods for Brain Tumor Detection in MR Images
}

\author{
K. Shankar, M. Ilayaraja, P. Deepalakshmi, S. Ramkumar, K. Sathesh Kumar
}

\begin{abstract}
In recent times, one of the perilous diseases which cause to increase the patient's death rate is a brain tumor. For diagnosing the tumor diseases from Magnetic Resonance Images (MRI), different classification methods have been analyzed. This paper presented an innovative method to diagnose brain tumor disease by using the classification process in MRI. From the input MR images, brain tumor image is classified by the supervised learning classifier i.e. Multi Kernel Support Vector machine (MKSVM). This model incorporates the extraction of feature and reduction process. All the MRI brain images are considered to extract some standard features and reduction reason dimensionality reduction that is Linear Discriminant Analysis (LDA) applied. Reason for this technique to the removal of multi-collinearity enhances the execution of the proposed model. Utilizing the feature vector attained out of the MRI images, SVM classifiers are utilized to image classification. The procedure comprises of two parts that are training stage as well as a testing stage. Parameters used to analyze the classified images as sensitivity, specificity, accuracy and so on. A cross-validation plot is adopted to enhance the generalization capability of the framework.
\end{abstract}

Keywords: Image Processing, MRI, Brian, Tumor Detection, Classification, LDA, MKSVM.

\section{INTRODUCTION}

The medical dataset image examination and training play an important role in the domain of pharmaceutical, in specific Noninvasive management and medical assessment. Medical imaging strategies and analysis apparatuses allow the two radiologists and specialists to enter at a particular analysis [1]. Generally, medical images are attained by various modalities like Computed Tomography (CT), X-rays, MRI, along with ultrasound imaging [2]. Nowadays MRI scanning device is utilized to scan the images onto films with the use of magnetic field [3]. Usually, the MRI brain images have both normal as

Revised Manuscript Received on December 15, 2019.

* Correspondence Author

K. Shankar*, School of Computing, Kalasalingam Academy of Research and Education, Krishnankoil, Virudhunagar, Tamil Nadu, India, shankar.k@klu.ac.in.

M. Ilayaraja, School of Computing, Kalasalingam Academy of Research and Education, Krishnankoil, Virudhunagar, Tamil Nadu, India, ilayaraja.m@klu.ac.in.

P. Deepalakshmi, School of Computing, Kalasalingam Academy of Research and Education, Krishnankoil, Virudhunagar, Tamil Nadu, India, deepa.kumar@klu.ac.in.

S. Ramkumar, School of Computing, Kalasalingam Academy of Research and Education, Krishnankoil, Virudhunagar, Tamil Nadu, India, ramkumar.dr12013@gmail.com.

K. Sathesh Kumar, School of Computing, Kalasalingam Academy of Research and Education, Krishnankoil, Virudhunagar, Tamil Nadu, India, sathesh.drl@gmail.com. well as abnormal images and the image differentiation is attained by image symmetric value at axial and coronal part of the brain image [4].

MRI distinguishes the radiated signals from the human brain i.e. normal and ab-normal tissues which give clear brain images. It has turned into a generally utilized strategy for fantastic medical imaging, [5] in specific, brain imaging where delicate non-invasiveness and tissue contrast are benefits [6]. Thusly, propel examination including MRI mind classification over the images is needed [7]. During the image training process, extraction of feature is a typical type of reduction of dimensionality. At the point while the information data to an method is too huge to ever be prepared and it is considered to [8] is despicably superfluous then the data information would be changed into a conservative portrayal set of features [9]. One among the striking features of the MR imaging is the fact that it is non-invasive in character. The elevation of aspects, which may be watched, is odd versus the parallel imaging modalities [10]. Then, select the optimally extracted features, these optimal features will provide the required information to feature reduction process [11]. Followed by the feature reduction, reduced features are used towards the image classifier as a support vector machine for training as well as testing the brain images [12]. By referring many re-search papers, there are several classifiers used for image classification, for example, K-Nearest Neighbor (KNN), SVM, Artificial Neural Network (ANN), Hidden Markov Model (HMM), Probabilistic Neural Network (PNN)[13]. Among these classifiers, SVM is a supervised learning classifier and perform depending on statistical learning hypothesis. In the study, we utilize MKSVM classifier which works on the principle of multiple kernels to automatically attain the classified brain image. When compared to other classifiers, the accuracy rate of the proposed MKSVM classifier is high and it is examined based on the training efficacy of the brain images [14].

\section{LITERATURE SURVEY}

MRI depending on Brain Tumor Analysis: Review on grade classification of brain tumor by GeethuMohana, M. Monica Subashini in 2018 [15]. Have proposed the MRI, the tumor might occur clear however physicians need quantification of tumor area for encouraging treatments to review the present patterns in both the classification and segmentation. 
The classification result relative to tumor based brain images with a goal over gliomas that incorporate astrocytoma.

The systems utilized for brain image feature extraction and brain tumor grading might be subjected to the standardardized medical imaging conventions are given.

In 2017 Sanjeev Kumar et al. [16] need from MR Images to recognize the brain tumor with aid of a hybrid method. This hybrid methodology consolidates DWT to be employed for feature extraction, GA for reducing the feature sets and SVM for arrangement of brain tumor. Images are derived from SICAS clinical Image Repository that classified images as circumspect or slander write. The simulation results exhibit that crossbreed method provide better implementation by improving precision and restricting the RMS botch in the examination with the superior in class frameworks in the comparative setting.

In 2017 Heba Mohsen et al. [17] the creator suggested the Deep Neural Network classifier for arranging a 66 brain MRIs and sarcoma dataset. The classifier were joined with DWT the genuine component extraction device and Principal Compo-nent Analysis (PCA) and the assessment of the execution was exceptionally incredible by and large the execution measures.

In 2017 Mahima and Padmavathi N B [18] a proficient classification strategy is presented for classification of brain neoplasm distinguished in MRI images. For training reason from Gray Level Co-occurrence Matrix (GLCM), 13 features are derived of MRI images. In addition to this, the accuracy of the proposed classifier is accessed through 10-overlay rotation computation plot."Two classifiers ANN and SVM MSE and computational time necessity.

\section{METHODOLOGY}

The main motive of the projected methodology is MRI image classification of the human brain; it's utilized a some phases of Preprocessing, reduction, Feature extraction and classification finally. At first, MRI images are assumed to features extraction process to derive the features (such as Texture, histogram and wavelet) of the brain images depending on approaches. The feature is first standardized to contain zero mean and unit change. A component determination procedure is then employed to pick a little course of action of feasible highlights for arrangement with a view to enhance the speculation capacity and the execution of the classifier. For lessening the computational (CPU) time as well as the classifying cost technique, reduction of feature is used that is Linear Discriminant Analysis (LDA). Extreme features are used for classification enhance computation time and increase storage memory. Finally, classifier phase towards the classification of the MRI images as benign, Normal and malignant, we utilized Multi Kernel SVM classifier, generally, classification issue having two phases which are training as well as testing stages; then the MKSVM is trained by the selected features of medical data. At the same time, in the testing stage, the result of classification process note whether the images comprise the brain tumor areas or the non-tumor areas. This approach appeared in figure 1, towards four categories e.g. glioblastoma, normal, metastatic had been looked at employing exactness, execution" measure

exceptional straightforwardness and minimum effort in both training and testing process in classifying MRI image and various kinds of domains and applications.

\section{A. MRI Images}

The traditional procedure in a medicine for cerebrum MR images order and tumor classification is a human assessment. It is a propelled medicinal imaging system that has wound up being an effective device in the examination of the human cerebrum. The rich data that MR images give about the fragile tissue life structures has drastically improved the nature of mind pathology conclusion and treatment, this Brian MRI image test showed up in figure 2. The grayscale image is otherwise called a power image. A variety of class pixel esteems decides drive esteems. For single and twofold clusters, values extend from 0 to 255 pixels [21]. The upside of this filtering as MRI especially significant for the checking and discovery of variations from the norm in sensitive tissue structures in the body like the ligament tissues.

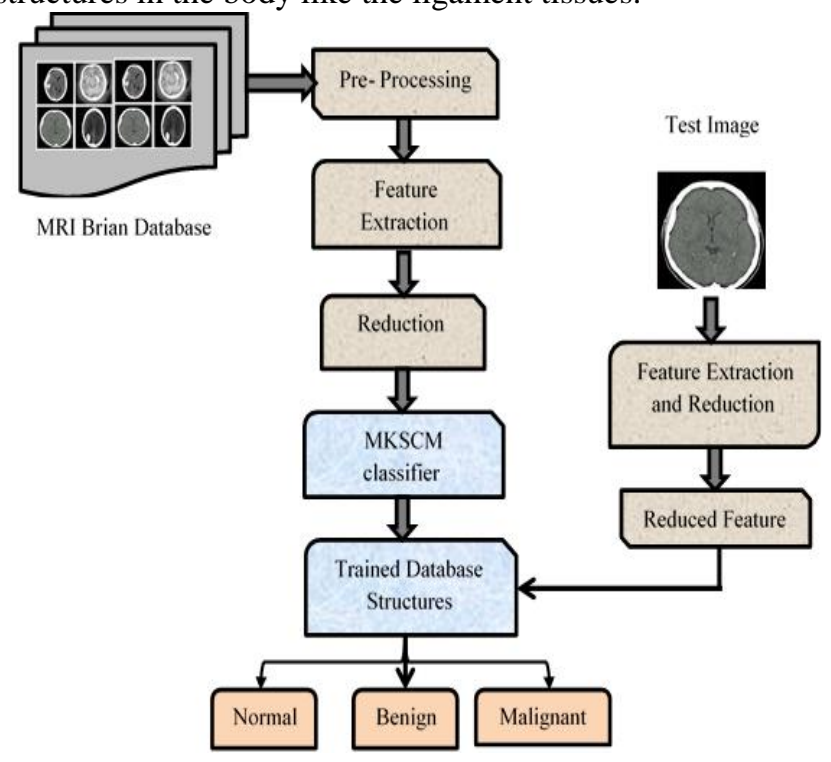

\section{Fig. 1.Overall structure for Projected MRI image classification.}

Most standard data blunder is obtained because of the abuse of shortenings, data passage botches, copy records, missing esteems. Inside this specific circumstance, one key research subject is the de-duplication issue which is the recognition and expulsion of copy records from a database. The exploration challenge is that databases contain both correct and vague copies. In the stage, the non-numerical data are cleared and procured the numerical dataset for proceeding with further.

\section{B. Filtering}

In image processing, filtering helps to remove noise from the considered MRI images. Clinical images are adulterated with various sorts of clatter when image obtaining. The versatile middle channel depends upon a trans-conductance comparator, in that submersion current might be changed to go about as a neighborhood weight administrator. 
This channel, when the image is noisy and goal pixels of the brain image adjacent pixel value is anywhere about 0's and 255 's through then supplant pixel in order to the intermediate value.

\section{Feature Extraction}

The contribution of extraction of feature in image processing is to derive the best subset of features from the MR Images. It reduces the feature set by selecting some required feature which can be used for effective classification. Features are employed as aid to classifiers that allocate it to the class that they are given. The objective of extraction of feature is to minimize the first information through computing positive features. This paper we have considered a few features, for example, Texture feature, Histogram Features, along with wavelet features.

Histogram Features.: The histogram of an image implies drive estimations of pixels. Histogram shows the count of pixels in an image at every power rate. Changing the power estimations of histogram of yield image roughly coordinates a foreordained histogram [22]. The histogram exhibits to us that the image contains just a small amount of the aggregate scope of gray dimensions. For this situation, there are 256 gray levels. It's having some normal features, for instance, "mean, imperativeness, skewness and kurtosis, standard deviation" conditions are depicted underneath.

Texture Features. Another type of feature extraction method is texture features. The abnormality of image is normally distribute in the MR brain image and the image textural structure of each class is extraordinary, which gives maximum classification accuracy. GLCM is a conventional technique of analyzing the surface that considering the pixel spatial relationship. The GLCM [23] function computing the recurrence of presence of the pixel pairs with the similar rates. In general, the required features are evaluated by the probability value of GLCM in the range of 22 feature sets ,among that we have considered a few features for our MRI Brian image classification process.

$$
G_{P_{i j}}=F_{i j} / \sum_{i, j=0}^{L-1} F_{i j}
$$

Above equation $F_{i j}$ as the occurrences frequency among two grey levels, $L$ - indicates a count of quantized grey levels, ${ }^{i}$ and ${ }^{j}$ for a applied displacement vector for certain window size.

Wavelet-based Features: It provides an appropriate basis to image processing as it is beneficial features. The DWT indicates a linear transformation which functions over a data vector which length is whole number energy of two, converting it to a numerical disparate identical length vector. It constitutes a gadget which segregates the data into differing recurrence fragment. In this feature extraction, two stages are there:

- Depending on particular recurrence subbands the natural images are decomposed.
- The disintegrated images at unmistakable recurrence subbands are evaluated utilizing various resolutions.

For including feature extraction and separate the image coefficients from MR images, a wavelet is used [24]. By then, the mean estimation of the coarse coefficients is evaluated by means of the normal coarse coefficient.

$$
\text { Coeff }\left[a_{t}\right]=\delta_{a_{t}}
$$

Where, the term $a$ indicates the mean of approximation coefficient. With the use of low pass filter, low recurrence brain images are screened out and in the same way, the high-frequency beat signals passing the remove recurrence by using the high pass filter.

\section{Feature Reduction: Linear Discriminant Analysis}

To minimize the primary data index by evaluating particular features or highlights which remembers one data design from other example. The general types of dimensionality reduction is LDA [20]. In the proposed LDA, input images are changed over to the independent feature set with a dimension, the diagrammatic representation appears in figure 2. Next to that feature reduction, dimensionality reduction of image feature vectors gives better classification results without loss of any data.

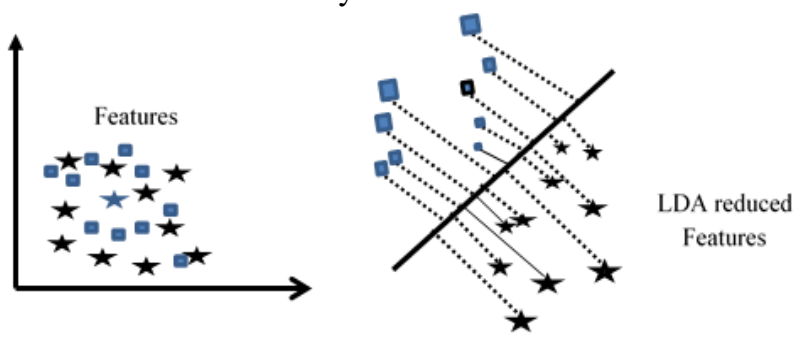

Fig. 2.LDA.

"The within-class distribute matrix is given as"

$$
M_{w}=\sum_{j=1}^{c} \sum_{i=1}^{N_{s}}\left(m_{i}^{j}-\alpha_{j}\right)\left(m_{i}^{j}-\alpha_{j}\right)^{T}
$$

Where ${ }^{c}$ is a number of classes, $m_{j}, N_{s}$ and $\alpha_{j}$ are class test, class tests count and mean of class. This standard tries to augment the ratio of the determinant of the between-class dissipates framework of the foreseen tests to the determinant of within-class lattice of the foreseen tests. Nonetheless, in multi-class LDA, the connections between the sets of classes are probably going to be not exactly the same as one set to another. From the lessen features into a classification procedure to classify the MRI images.

\section{E. Classification}

The SVM method tries to search the optimal distinguishing hyperplane among the classes through aiming over the training cases which are put at the class edge descriptors. These training cases are known as the SVM vectors. The training cases other than the support vectors are disposed of vectors. SVM describes among two classes through building up a hyperplane in high-dimensional segment space that might be utilized for characterization demonstrated up.

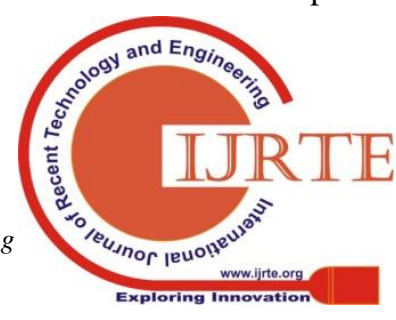


SVM describes among two classes through building up a hyperplane in high-dimensional segment space that can be utilized for characterization appeared. SVM [19] is a classifier, that depends on various kernel strategies. Our proposed classification process multi kernels are considered RBF, linear kernel and MLP, is cross breed to choose the average hyperplane of SVM process.

$$
M_{k 1} \Rightarrow \exp \left(-\frac{1}{2}\left\|h_{1}-h_{2}\right\|^{2}\right)=\sum_{j=0}^{\infty} \frac{\left(h_{1}{ }^{T}, h_{2}\right)^{j}}{j !} \exp \left(-\frac{1}{2}\left\|h_{1}\right\|^{2}\right) \exp \left(-\frac{1}{2}\left\|h_{2}\right\|^{2}\right)
$$

While the value of the RBF kernel reduces through detachment and arrangement between zeros. Here $K\left(h_{1}, h_{2}\right)$ represents the inner result of two vectors prompted in the feature space by the info vector.

Multi-Layer Perceptron function (MLP): The MLP Kernel obtains its basis from the field of the Neural Networks, where the bipolar sigmoid utility is consistently involved as an instigation utility for artificial neurons. In the equation give beneath, $h_{1}$ and $h_{2}$ values are acquired as +1 and -1 correspondingly.

$$
M_{k 2} \Rightarrow k\left(h_{1}, h_{2}\right)=\tanh \left(p 1 \times\left(h_{1} \times h_{2}{ }^{\prime}\right)+p 2\right)
$$

Linear Kernel: In the linear kernel, the normal and abnormal classes are linearly separable, which infers that it is conceivable to situate no short of what one hyperplane characterized through a vector $w$ with a bias, that can isolate the image classes with zero error.

$$
M_{k 3} \Rightarrow K\left(h, h_{i}\right)=h . h_{i}
$$

Multi kernels for classification: For the consideration of the combination of three kernel function in SVM process, the hybrid kernel function classifies the images as "Normal or benign or malignant". Consider offer training set, when there may exist numerous hyperplane that amplifies the isolating edge among the two classes, it depends on the hyperplane that boosts the isolating edge between the two classes.

$$
M_{k}=\frac{M_{k 1}+M_{k 2}+M_{k 3}}{3}
$$

The average of three kernels gives the optimal outcome as signified in above equation. Based on the training dataset, image class is recognized with aid of image labels. In the testing phase, classified data are analyzed and unlabeled data are given on the basis of the unlabeled point. From the testing, all normal have been classified effectively yet not for abnormal ones [18].

Radial Basis Function (RBF): RBF network can be misused to discover a set weight for a bend not too bad inconvenience. The weights are in prevalent dimensional gap than the innovative data. Learning is equal to discovery an outside in high dimensional gap that supplies the greatest fit to preparation data.

\section{RESULT AND DISCUSSION}

The presented MRI brain tumor image classification model is implemented in MATLAB 2016 platform with the system setup as an i5 processor with 4GB RAM. Here, standard MRI database is considered for the tumor image classification process. The performance of MKSVM classifier is analyzed and compared with the existing classifiers SVM, NN, and KNN etc., based on different measures including its accuracy.

\section{A. Database Description}

The MRI image dataset that we have used in our proposed tumor detection procedure is taken from the sources freely available. This image data set contains brain MRI images which include "normal, benign and malignant". This database gathered out of "Whole Brain Atlas", its database comprise of 104 instances (Figure 3) in the axial plane of T2-weighted MR brain data and $255^{*} 255$ in-plane resolutions.
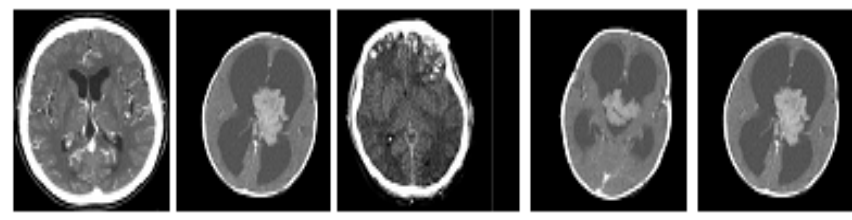

Fig. 3.Sample Database Images

Table 1 demonstrates the results of proposed MKSVM classifier. In the analysis, the input MRI brain image is classified as three kinds namely, normal or benign or malignant. Proposed MKSVM compared to more literature classifiers to demonstrate our model as best. The matrix-based calculation increases the performance measures, the projected work is, and primarily, it expels sensitivity to primary values of bunch focuses utilizing hybrid evolutionary classification method second, color feature concern and surface features for grouping MR brain tumor datasets to enhance classification proficiency [26-29]. Finally comparative analysis shows in figure 4, our proposed model compared with other existing technique Optimal Deep Neural Network (ODNN) [25], ANN and KNN techniques. The variation in accuracy analysis is observed based on the kernel function of MKSVM classifier.

TABLE-I: Proposed MKSVM - Test Results for MRI Classification.

\begin{tabular}{|c|c|c|c|c|}
\hline MRI image & Type & Accuracy & Sensitivity & Specificity \\
\hline & & & & \\
\hline
\end{tabular}




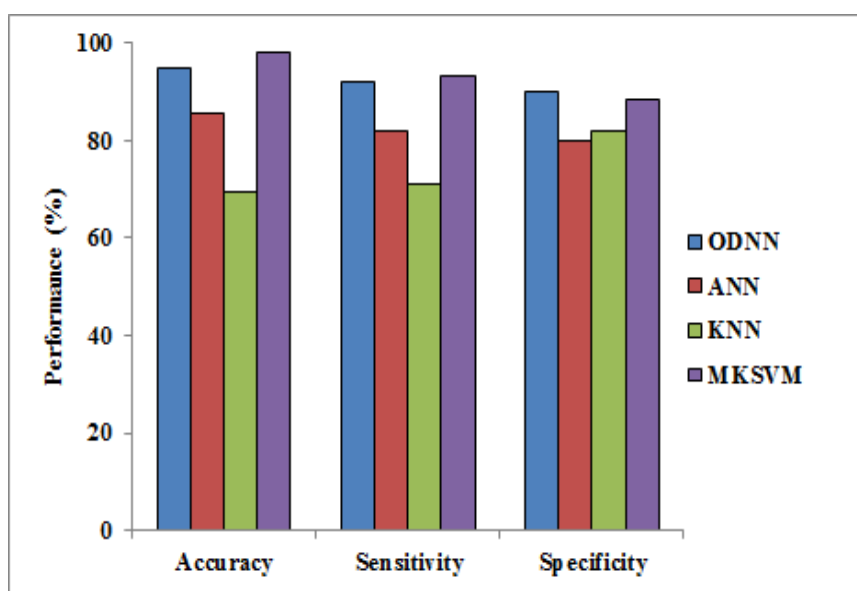

Fig. 4.Comparative analysis

\section{CONCLUSION}

Brains Tumor MRI classification with include choice and extraction have been done in the past with compelled accomplishment. "The proposed MKSVM with feature reduction to demonstrate the better classification compared with others; Brain MRI Images keeping in mind the end goal to classification of brain tumor in benign or malignant". Robust brain tumor detection method lessens the physical labeling time and avoids the human mistake. By a machine learning technique, we achieved better accuracy in the process of classifying abnormal and normal brain image. The results conclude that the proposed MKSVM is more effective by means of sensitivity, accuracy, and specificity as $96.2 \%$, 94.56 and $94.2 \%$. While recognizing the tumor-affected parts in the MRI Brain images, the accuracy level of MKSVM algorithm is very efficient. Also, the performance measures of this classifier show its advantages: it is rapid, cheap, easier to work and non-invasive.

\section{REFERENCES}

[1] Zhang, Y., Dong, Z., Wu, L. and Wang, S., 2011. A hybrid method for MRI brain image classification. Expert Systems with Applications, 38(8), pp.10049-10053.

[2] Gupta, T., Manocha, P., Gandhi, T.K., Gupta, R.K. and Panigrahi, B.K., 2017. Tumor Classification and Segmentation of MR Brain Images. arXiv preprint ar-Xiv:1710.11309,pp.1-20.

[3] Kanmani, P. and Marikkannu, P., 2018. MRI Brain Images Classification: A Multi-Level Threshold Based Region Optimization Technique. Journal of medical systems, 42(4), p.62.

[4] Suthar, M.H., and Gupta, M., 2017. A NOVEL APPROACH FOR MRI BRAIN IMAGE CLASSIFICATION AND DETECTION. Journal of Engineering and Technology, Vol.4, No.1,pp.3260-3267.

[5] Vani, V. and Geetha, M.K., 2016. Automatic Tumor Classification of Brain MRI Im-ages. International Journal of Computer Sciences and Engineering, 4(10), pp.144-151.

[6] Bahadure, N.B., Ray, A.K. and Thethi, H.P., 2017. Image analysis for MRI based brain tumor detection and feature extraction using biologically inspired BWT and SVM. International journal of biomedical imaging, 2017.pp,.1-13.

[7] P.B.Nikam and V.D.Shinde, 2013 MRI Brain Image Classification and Detection Us-ing Distance Classifier Method in Image Processing, Journal of Engineering Research \& Technology, Vol.2, No.6,pp.1980-1985.

[8] El-Dahshan, E.S.A., Hosny, T. and Salem, A.B.M., 2010. Hybrid intelligent tech-niques for MRI brain images classification. Digital Signal Processing, 20(2), pp.433-441.

[9] Krishnan, G.S., Sivanarulselvan, K. and Betty, P., 2016. Survey On Brain Tumour Detection And Classification Using Image Processing.,
Journal Of Computer Science And Information Systems, Vol.2, No.1,pp.1-8.

[10] Sahu, O., Anand, V., Kanhangad, V. and Pachori, R.B., 2015. Classification of mag-netic resonance brain images using bi-dimensional empirical mode decomposition and autoregressive model. Biomedical Engineering Letters, 5(4), pp.311-320.

[11] Abdullah, N., Ngah, U.K. and Aziz, S.A., 2011, May. Image classification of brain MRI using support vector machine. In Imaging Systems and Techniques (IST), 2011 IEEE International Conference on (pp. 242-247). IEEE.

[12] Nandpuru, H.B., Salankar, S.S. and Bora, V.R., 2014, March. MRI brain cancer clas-sification using support vector machine. In Electrical, Electronics and Computer Science (SCEECS), 2014 IEEE Students' Conference on (pp. 1-6). IEEE.

[13] Ibrahim, W.H., Osman, A.A.A. and Mohamed, Y.I., 2013, August. MRI brain image classification using neural networks. In Computing, Electrical and Electronics Engineering (ICCEEE), 2013 International Conference on (pp. 253-258). IEEE.

[14] Sonavane, R., Sonar, P. and Sutar, S., 2017, May. Classification of MRI brain tumor and mammogram images using learning vector quantization neural network. In Sensing, Signal Processing and Security (ICSSS), 2017 Third International Conference on (pp. 301-307). IEEE.

[15] Mohan, G. and Subashini, M.M., 2018. MRI based medical image analysis: Survey on brain tumor grade classification. Biomedical Signal Processing and Control, 39, pp.139-161.

[16] Kumar, S., Dabas, C. and Godara, S., 2017. Classification of Brain MRI Tumor Im-ages: A Hybrid Approach. Procedia Computer Science, 122, pp.510-517

[17] Mohsen, H., El-Dahshan, E.S.A., El-Horbaty, E.S.M., and Salem, A.B.M., 2017. Classification using Deep Learning Neural Networks for Brain Tumors. Future Computing and Informatics Journal., pp.1-8.

[18] Padmavathi, N.B., 2017, July. Comparative study of kernel SVM and ANN classifiers for brain neoplasm classification. In Intelligent Computing, Instrumentation and Control Technologies (ICICICT), 2017 International Conference on (pp. 469-473). IEEE.

[19] Al-Badarneh, A., Najadat, H., and Alraziqi, A.M., 2012, August. A classifier to detect tumor disease in MRI brain images. In Proceedings of the 2012 International Conference on Advances in Social Networks Analysis and Mining (ASONAM 2012) (pp. 784-787). IEEE Computer Society.

[20] Ren, H. and Chang, Y.L., 2005, November. Feature extraction with modified Fisher's linear discriminant analysis. In Chemical and Biological Standoff Detection III (Vol. 5995, p. 599506). International Society for Optics and Photonics.

[21] Sheejakumari, V. and Gomathi, A., 2012. Healthy and pathological tissues classification in MRI brain images using hybrid genetic algorithm-neural network (HGANN) approach. European Journal of Scientific Research, 87(2), pp.212-226.

[22] Vinotha, K., 2014. Brain tumor detection and classification using histogram equalization and fuzzy support vector machine approach International journal of engineering and computer science, 3(05), PP.5823-5827.

[23] Zulpe, N. and Pawar, V., 2012. GLCM textural features for brain tumor classification. IJCSI International Journal of Computer Science Issues, 9(3), pp.354-359.

[24] Kumar, C.M., and Kumar, S.D., 2007. Wavelet-Based Feature Extraction Scheme of Electroencephalogram. Journal of Innovative Research in Science, Engineering, and Technology,3(1),pp.908-913. Lakshmanaprabu, S.K., Mohanty, S.N., Shankar, K., Arunkumar, N. and Ramirez, G., 2019. Optimal deep learning model for classification of lung cancer on CT images. Future Generation Computer Systems, 92, pp.374-382

[25] Elhoseny, M., Shankar, K., Lakshmanaprabu, S. K., Maseleno, A., \& Arunkumar, N. (2018). Hybrid optimization with cryptography encryption for medical image security in Internet of Things. Neural Computing and Applications, 1-15 https://doi.org/10.1007/s00521-018-3801-X

[26] Shankar, K., Elhoseny, M., Chelvi, E. D., Lakshmanaprabu, S. K., \& Wu, W. (2018). An Efficient Optimal Key Based Chaos Function for Medical Image Security. IEEE Access, 6, 77145-77154.

[27] Avudaiappan, T., Balasubramanian, R., Pandiyan, S. S., Saravanan, M., Lakshmanaprabu, S. K., \& Shankar, K. (2018). Medical image security using dual encryption with oppositional based optimization algorithm. Journal of medical systems, 42(11), 208.

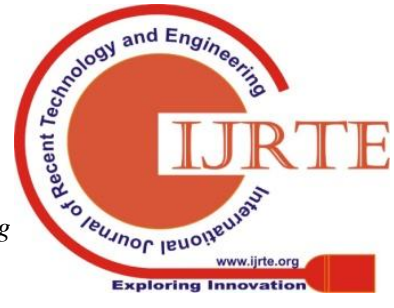


Effective MKSVM Classifier with LDA Methods for Brain Tumor Detection in MR Images

[28] Shankar, K., Elhoseny, M., Lakshmanaprabu, S. K., Ilayaraja, M., Vidhyavathi, R. M., Elsoud, M. A., \& Alkhambashi, M. (2018). Optimal feature level fusion based ANFIS classifier for brain MRI image classification. Concurrency Computat Pract Exper, e4887. 